Proceedings of the

International Geometry Center

Vol. 12, no. 2 (2019) pp. 43-61

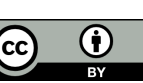

\author{
-
}

.

\title{
On fractal properties of Weierstrass-type functions
}

\author{
Claire David
}

\begin{abstract}
In the sequel, starting from the classical Weierstrass function defined, for any real number $x$, by $\mathcal{W}(x)=\sum_{n=0}^{+\infty} \lambda^{n} \cos \left(2 \pi N_{b}^{n} x\right)$, where $\lambda$ and $N_{b}$ are two real numbers such that $0<\lambda<1, N_{b} \in \mathbb{N}$ and $\lambda N_{b}>1$, we highlight intrinsic properties of curious maps which happen to constitute a new class of iterated function system. Those properties are all the more interesting, in so far as they can be directly linked to the computation of the box dimension of the curve, and to the proof of the non-differentiabilty of Weierstrass type functions.
\end{abstract}

Анотація. Метою даної роботи є узагальнення попередніх результатів автора про класичну функцію Вейерштрасса та її графік. Його можна отримати як границю послідовності префракталів, тобто графів, отриманих за допомогою ітераційної системи функцій, які, як правило, не є стискаючими відображеннями. Натомість вони мають в деякому сенсі еквівалентну властивість до стискаючих відображень, оскільки на кожному етапі ітераційного процесу, який дає змогу отримати префрактали, вони зменшують двовимірні міри Лебега заданої послідовності прямокутників, що покривають криву. Такі системи функцій відіграють певну роль на першому кроці процесу побудови підкови Смейла. Вони можуть бути використані для доведення недиференційованості функції Вейєрштрасса та обчислення bох-розмірності її графіка, а також для побудови більш широких класів неперервних, але ніде не диференційовних функцій. Останне питання ми вивчатимемо в подальших роботах.

2010 Mathematics Subject Classification: 37F20, 28A80, 05C63

Keywords: Weierstrass function; non-differentiability; iterative function systems DOI: http://dx.doi.org/10.15673/tmgc.v12i2.1485 


\section{INTRODUCTION}

In his seminal paper of 1981, J. E. Hutchinson [8] introduces, for the first time, what will be later qualified of "iterated function system" (I.F.S.), as a finite set of contraction maps, each defined on a compact metric set $K$ of the euclidean space $\mathbb{R}^{d}, d \in \mathbb{N}^{\star}$ :

$$
\mathcal{S}=\left\{T_{1}, \ldots, T_{N}\right\}, \quad N \in \mathbb{N}^{\star}
$$

where $\mathbb{N}^{\star}$ denotes the set of strictly positive integers, such that

$$
K=\bigcup_{i=1}^{N} T_{i}(K)
$$

The compact set $K$ is then said to be "invariant" with respect to the set $\mathcal{S}$ (one often refer to this result as the "Gluing Lemma").

A prequel occurence of such maps, under the form of similitudes, can already be found in the Mandelbrot books of 1977 [11], [12].

Hutchinson's novelty is to consider not the compact $K$ itself, but the set $\mathcal{S}$, which arises naturally, in so far as the invariant compact $K$ is fully determined by the set $\mathcal{S}$, and, interestingly, is also the limit of a sequence of pre-fractal graphs that can be built, in an iterative way, thanks to the maps that constitute the set $\mathcal{S}$.

Following this work, iterated function systems were taken up and even more developed by M. F. Barnsley et al. [2], as "a unified way of generating and classifying a broad class of fractals". As explained by the authors, fractals were "traditionally viewed as being produced by a process of successive microscopic refinement taken to the limit", which, of course, makes sense with the geometric representation one may have of fractal sets, since, when looking at smaller and smaller scales, one finds, again and again, the same form. Of course, at stake are specific and classical types of fractals, as Sierpiński gaskets, dragon curves, Cantor sets, Julia curves, etc. For M. F. Barnsley and S. Demko, those fractals are to be seen as the attractors of iterated function systems, which, of course, joins the approach of J. E. Hutchinson.

M. F. Barnsley and S. Demko place themselves in a probabilistic approach. Given still a compact metric space $K$, the related Banach space $C(K)$ of real-valued functions defined on $K$, with respect to the norm

$$
f \in C(K) \mapsto\|f\|_{\infty}=\max \{|f(x)|, x \in K\}
$$

and a finite collection

$$
w=\left\{w_{1}, \ldots, w_{N}\right\}, \quad N \in \mathbb{N}^{\star}
$$

of Borel measurable functions from $K$ to $K$, they define the set $\{K, w\}$ as an iterated function system if and only if there exists an associated set of 
positive real numbers

$$
\left\{p_{1}, \ldots, p_{N}\right\}, \quad \forall i \in\{1, \ldots, N\}: p_{i}>0, \quad \sum_{i=1}^{N} p_{i}=1
$$

such that the operator $T$ on $C(K)$, given, for any $f$ of $C(K)$, by

$$
\forall x \in K: \quad T(f)(x)=\sum_{i=1}^{N} p_{i}\left(f \circ w_{i}\right)(x)
$$

has the property:

$$
T(C(K)) \subset C(K) .
$$

Treating $w$ as a set-valued function, through

$$
\forall x \in K: \quad w(x)=\left\{w_{1}(x), \ldots, w_{N}(x)\right\}
$$

they then naturally introduce, for the i.f.s. $\{K, w\}$, and a given $x$ of $K$, the related attractor

$$
\mathcal{A}(x)=\lim _{n \rightarrow+\infty} w^{\circ^{n}}(x)
$$

in the sense:

$$
\lim _{n \rightarrow+\infty}\left\|w^{\circ^{n}}(x)-\mathcal{A}(x)\right\|_{\infty}=0 .
$$

Classical fractal sets as, for instance, the Sierpiński Gasket, fit this definition.

In our previous work on the Weierstrass curve [4], which, as exposed, for instance, by A. S. Besicovitch and H. D. Ursell [3], or, a few years later, by B. Mandelbrot [11], bears fractal properties, we showed that the curve could be obtained by means of a sequence a graphs $\left(\Gamma_{\mathcal{W}_{m}}\right)_{m \in \mathbb{N}}$, that approximate the studied one. This is done using a family of nonlinear $C^{\infty}$ maps from $\mathbb{R}^{2}$ to $\mathbb{R}^{2}$, which happen not to be contractions, in the aforementioned classical sense. The nonlinearity does not enable one to resort to the probabilistic approach of M. F. Barnsley and S. Demko, since there does not exist a constant associated set of probabilities. Yet, even if they are not contractions, our maps bear what can be viewed as an equivalent property, since, at each step of the iterative process, they reduce the two-dimensional Lebesgue measures of a given sequence of rectangles covering the curve. This is due to the fact that they correspond, in a sense, to the composition of a contraction of ratio $r_{x}$ in the horizontal direction, and a dilatation of factor $r_{y}$ in the vertical one, with

$$
r_{x} r_{y}<1
$$

Such maps are considered in the book of Robert L. Devaney [6], where they play a part in the first step of the horseshoe map process introduced by Stephen Smale. 
The Weierstrass curve is invariant with respect to the set of those maps, which makes it possible to dispose of an equivalent result of the Gluing Lemma. But what deserves to be enlightened, in our case, is that the intrinsic properties of those curious maps make them all the more interesting, in so far as they can be directly linked to the computation of the box dimension of the curve, and to the proof of the non-differentiabilty of the Weierstrass function, as shown in [5]. All the more is the generalization to a broader class of applications that could, then, enable one to build everywhere continuous, though nowhere differentiable, functions, as we will expose it in the sequel.

\section{The Case of the Weierstrass function}

Notation 1.1. In the following, $\lambda$ and $b$ are two real numbers such that:

$$
0<\lambda<1, \quad b=N_{b} \in \mathbb{N}, \quad \lambda N_{b}>1 .
$$

We deliberatly made the choice to introduce the notation $N_{b}$ which replaces the initial $b$, in so far as, to the origins, $b$ is any real number, whereas we deal with the specific case of a natural integer that we consequently choose to denote by $N_{b}$, as an echo to the initial $b$.

The Weierstrass function, introduced in 1875 by K. Weierstrass [13], known as one of these so-called pathological mathematical objects, continuous everywhere, while nowhere differentiable, is the sum of the uniformly convergent trigonometric series, defined, for any real number $x$, by:

$$
\mathcal{W}(x)=\sum_{n=0}^{+\infty} \lambda^{n} \cos \left(2 \pi N_{b}^{n} x\right)
$$

Definition 1.2. (Weierstrass Curve). We will call Weierstrass Curve the restriction to $[0,1) \times \mathbb{R}$, of the graph of the Weierstrass function, and denote it by $\Gamma_{\mathcal{W}}$.

Theoretical study. We place ourselves, in the following, in the euclidian plane of dimension 2, referred to a direct orthonormal frame. The usual Cartesian coordinates are $(x, y)$.

Property 1.3. (Periodic properties of the Weierstrass function). For any real number $x$ :

$$
\mathcal{W}(x+1)=\sum_{n=0}^{+\infty} \lambda^{n} \cos \left(2 \pi N_{b}^{n} x+2 \pi N_{b}^{n}\right)=\sum_{n=0}^{+\infty} \lambda^{n} \cos \left(2 \pi N_{b}^{n} x\right)=\mathcal{W}(x) .
$$

The study of the Weierstrass function can be restricted to the interval $[0,1)$.

By following the method developed by J. Kigami [10], we approximate the restriction $\Gamma_{\mathcal{W}}$ to $[0,1) \times \mathbb{R}$, of the Weierstrass Curve, by a sequence of 
graphs, built through an iterative process. To this purpose, we introduce the iterated function system of the family of $C^{\infty}$ maps from $\mathbb{R}^{2}$ to $\mathbb{R}^{2}$ :

$$
\left\{T_{0}, \ldots, T_{N_{b}-1}\right\}
$$

where, for any integer $i$ belonging to $\left\{0, \ldots, N_{b}-1\right\}$ and any $(x, y)$ of $\mathbb{R}^{2}$ :

$$
T_{i}(x, y)=\left(\frac{x+i}{N_{b}}, \lambda y+\cos \left(2 \pi \frac{x+i}{N_{b}}\right)\right) .
$$

Property 1.4. [4]. $\Gamma_{\mathcal{W}}=\bigcup_{i=0}^{N_{b}-1} T_{i}\left(\Gamma_{\mathcal{W}}\right)$.

Definition 1.5. (Word on the graph $\Gamma_{\mathcal{W}}$ ). Let $m$ be a strictly positive integer. We will call number-letter any integer $\mathcal{M}_{i}$ of $\left\{0, \ldots, N_{b}-1\right\}$, and word of length $|\mathcal{M}|=m$, on the graph $\Gamma_{\mathcal{W}}$, any set of number-letters of the form:

$$
\mathcal{M}=\left(\mathcal{M}_{1}, \ldots, \mathcal{M}_{m}\right)
$$

We will write:

$$
T_{\mathcal{M}}=T_{\mathcal{M}_{1}} \circ \cdots \circ T_{\mathcal{M}_{m}} .
$$

Definition 1.6. For any integer $i$ belonging to $\left\{0, \ldots, N_{b}-1\right\}$, let us denote by:

$$
P_{i}=\left(x_{i}, y_{i}\right)=\left(\frac{i}{N_{b}-1}, \frac{1}{1-\lambda} \cos \left(\frac{2 \pi i}{N_{b}-1}\right)\right)
$$

the fixed point of the map $T_{i}$.

We will denote by $V_{0}$ the ordered set (according to increasing abscissa), of the points:

$$
\left\{P_{0}, \ldots, P_{N_{b}-1}\right\}
$$

since for any $i$ of $\left\{0, \ldots, N_{b}-2\right\}$ :

$$
x_{i} \leqslant x_{i+1}
$$

The set of points $V_{0}$, where, for any $i$ of $\left\{0, \ldots, N_{b}-2\right\}$, the point $P_{i}$ is linked to the point $P_{i+1}$, constitutes an oriented graph (according to increasing abscissa), which we will denote by $\Gamma_{\mathcal{W}_{0}}$. In turn, $V_{0}$ is called the set of vertices of the graph $\Gamma_{\mathcal{W}_{0}}$.

For any natural integer $m$, we set:

$$
V_{m}=\bigcup_{i=0}^{N_{b}-1} T_{i}\left(V_{m-1}\right) .
$$

The set of points $V_{m}$, where two consecutive points are linked, is an oriented graph (according to increasing abscissa), which we will denote by $\Gamma_{\mathcal{W}_{m}}$. Again $V_{m}$ is called the set of vertices of the graph $\Gamma_{\mathcal{W}_{m}}$. In what 
follows we will denote by $\mathcal{N}_{m}^{\mathcal{S}}$ the number of vertices of the graph $\Gamma_{\mathcal{W}_{m}}$, and write:

$$
V_{m}=\left\{\mathcal{S}_{0}^{m}, \mathcal{S}_{1}^{m}, \ldots, \mathcal{S}_{\mathcal{N}_{m}^{\mathcal{S}}-1}\right\}
$$

Property 1.7. For any natural integer $m$ :

$$
V_{m} \subset V_{m+1} \text {. }
$$

Property 1.8. For any integer $i$ belonging to $\left\{0, \ldots, N_{b}-2\right\}$ :

$$
T_{i}\left(P_{N_{b}-1}\right)=T_{i+1}\left(P_{0}\right) \text {. }
$$

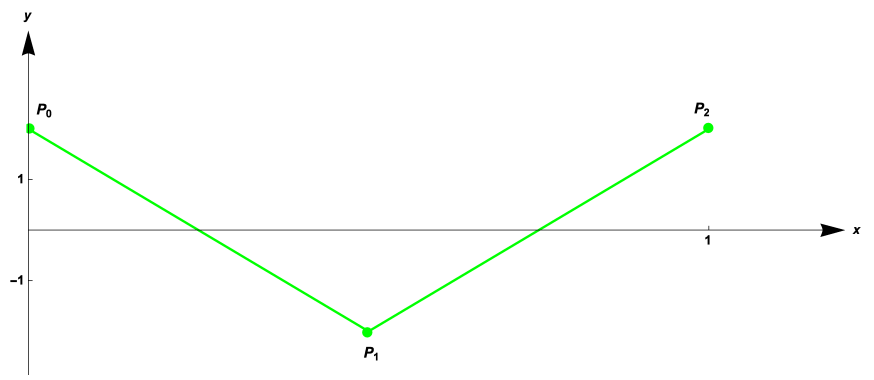

Figure 1.1. Fixed points $P_{0}, P_{1}, P_{2}$, and the graph $\Gamma_{\mathcal{W}_{0}}$, in the case when $\lambda=\frac{1}{2}$ and $N_{b}=3$.

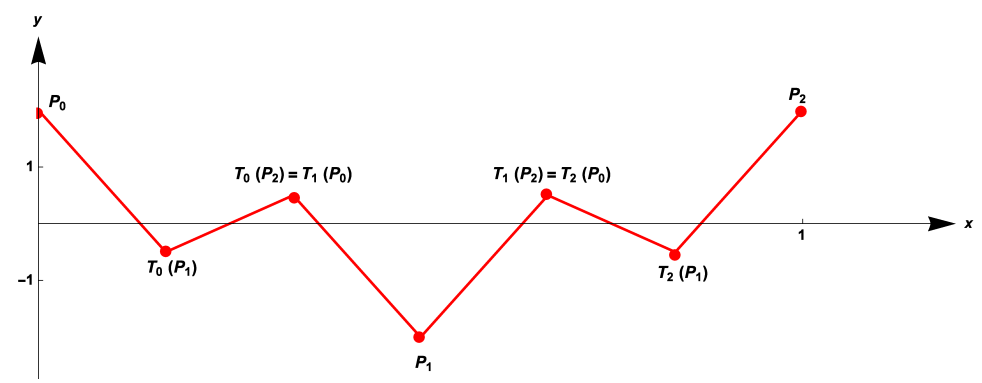

Figure 1.2. Graph $\Gamma_{\mathcal{W}_{1}}$, in the case when $\lambda=\frac{1}{2}, N_{b}=3$, $T_{0}\left(P_{2}\right)=T_{1}\left(P_{0}\right)$, and $T_{1}\left(P_{2}\right)=T_{2}\left(P_{1}\right)$.

Definition 1.9. (Vertices of the graph $\Gamma_{\mathcal{W}}$ ). Two points $X$ and $Y$ of $\Gamma_{\mathcal{W}}$ will be called vertices of the graph $\Gamma_{\mathcal{W}}$ if there exists a natural integer $m$ such that:

$$
(X, Y) \in V_{m}^{2}
$$

Definition 1.10. (Consecutive vertices on the graph $\Gamma_{\mathcal{W}}$ ). Two points $X$ and $Y$ of $\Gamma_{\mathcal{W}}$ will be called consecutive vertices of the graph $\Gamma_{\mathcal{W}}$ if there exist a natural integer $m$, and an integer $j$ of $\left\{0, \ldots, N_{b}-2\right\}$, such that:

$$
\left\{\begin{array}{l}
X=\left(T_{i_{1}} \circ \ldots \circ T_{i_{m}}\right)\left(P_{j}\right) \\
Y=\left(T_{i_{1}} \circ \ldots \circ T_{i_{m}}\right)\left(P_{j+1}\right)
\end{array} \quad\left\{i_{1}, \ldots, i_{m}\right\} \in\left\{0, \ldots, N_{b}-1\right\}^{m}\right.
$$




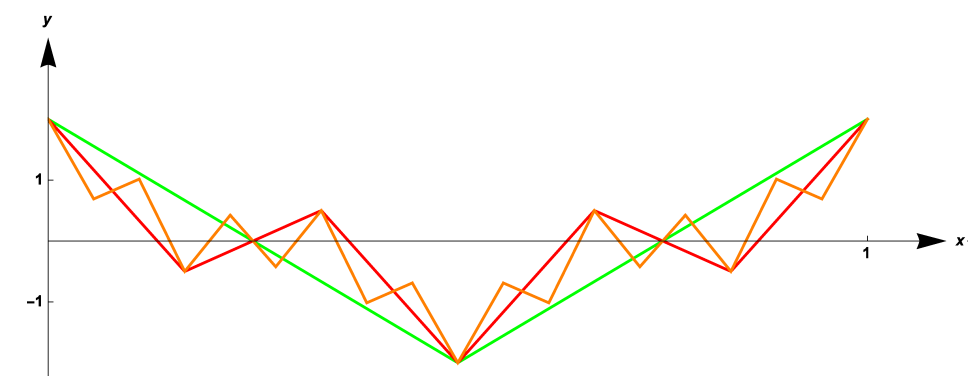

Figure 1.3. Graphs $\Gamma_{\mathcal{W}_{0}}$ (in green), $\Gamma_{\mathcal{W}_{1}}$ (in red), $\Gamma_{\mathcal{W}_{2}}$ (in orange), $\Gamma_{\mathcal{W}}$ (in cyan), in the case where $\lambda=\frac{1}{2}$ and $N_{b}=3$.

or:

$$
X=\left(T_{i_{1}} \circ T_{i_{2}} \circ \ldots \circ T_{i_{m}}\right)\left(P_{N_{b}-1}\right), \quad Y=\left(T_{i_{1}+1} \circ T_{i_{2}} \ldots \circ T_{i_{m}}\right)\left(P_{0}\right) .
$$

Property 1.11. The set $\bigcup_{m \in \mathbb{N}} V_{m}$ is dense in $\Gamma_{\mathcal{W}}$.

Definition 1.12. (Edge relation, on the graph $\Gamma_{\mathcal{W}}$ ). Given a natural integer $m$, two points $X$ and $Y$ of $\Gamma_{\mathcal{W}_{m}}$ will be called adjacent if and only if $X$ and $Y$ are two consecutive vertices of $\Gamma_{\mathcal{W}_{m}}$. We will write:

$$
X \underset{m}{\sim} Y
$$

This edge relation ensures the existence of a word $\mathcal{M}=\left(\mathcal{M}_{1}, \ldots, \mathcal{M}_{m}\right)$ of length $m$, such that $X$ and $Y$ both belong to the iterate:

$$
T_{\mathcal{M}} V_{0}=\left(T_{\mathcal{M}_{1}} \circ \cdots \circ T_{\mathcal{M}_{m}}\right) V_{0}
$$

Given two points $X$ and $Y$ of the graph $\Gamma_{\mathcal{W}}$, we will say that $X$ and $Y$ are adjacent if and only if there exists a natural integer $m$ such that:

$$
X \underset{m}{\sim} Y
$$

Proposition 1.13 (Adresses, on the Weierstrass Curve). Given a strictly positive integer $m$, and a word $\mathcal{M}=\left(\mathcal{M}_{1}, \ldots, \mathcal{M}_{m}\right)$ of length $m \in \mathbb{N}^{\star}$, on the graph $\Gamma_{\mathcal{W}_{m}}$, for any integer $j$ of $\left\{1, \ldots, N_{b}-2\right\}$, any $X=T_{\mathcal{M}}\left(P_{j}\right)$ of $V_{m} \backslash V_{0}$, i.e. distinct from one of the $N_{b}$ fixed point $P_{i},\left(0 \leqslant i \leqslant N_{b}-1\right)$, has exactly two adjacent vertices, given by:

$$
T_{\mathcal{M}}\left(P_{j+1}\right) \quad \text { and } \quad T_{\mathcal{M}}\left(P_{j-1}\right)
$$

where:

$$
T_{\mathcal{M}}=T_{\mathcal{M}_{1}} \circ \cdots \circ T_{\mathcal{M}_{m}}
$$

By convention, the adjacent vertices of $T_{\mathcal{M}}\left(P_{0}\right)$ are $T_{\mathcal{M}}\left(P_{1}\right)$ and $T_{\mathcal{M}}\left(P_{N_{b}-1}\right)$, those of $T_{\mathcal{M}}\left(P_{N_{b}-1}\right), T_{\mathcal{M}}\left(P_{N_{b}-2}\right)$ and $T_{\mathcal{M}}\left(P_{0}\right)$. 
Notation 1.14. For any integer $j$ belonging to $\left\{0, \ldots, N_{b}-1\right\}$, any natural integer $m$, and any word $\mathcal{M}$ of length $m$, we set:

$$
\begin{aligned}
T_{\mathcal{M}}\left(P_{j}\right) & =\left(x\left(T_{\mathcal{M}}\left(P_{j}\right)\right), y\left(T_{\mathcal{M}}\left(P_{j}\right)\right)\right), \\
L_{m} & =x\left(T_{\mathcal{M}}\left(P_{j+1}\right)\right)-x\left(T_{\mathcal{M}}\left(P_{j}\right)\right)=\frac{1}{\left(N_{b}-1\right) N_{b}^{m}} \\
h_{j, m} & =y\left(T_{\mathcal{M}}\left(P_{j+1}\right)\right)-y\left(T_{\mathcal{M}}\left(P_{j}\right)\right) .
\end{aligned}
$$

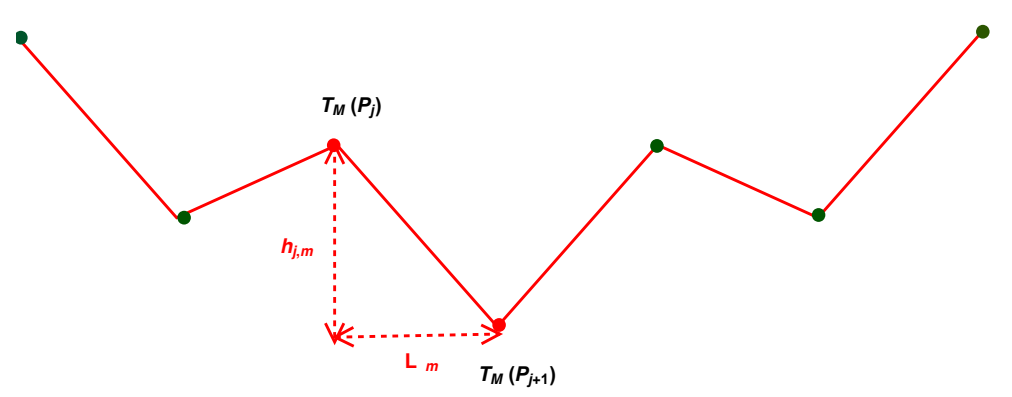

Notation 1.15. We will denote by

$$
D_{\mathcal{W}}=2+\frac{\ln \lambda}{\ln N_{b}}
$$

the Hausdorff dimension of $\Gamma_{\mathcal{W}}$, see [1], [9].

Theorem 1.16 (An upper bound and a lower bound, for the box-dimension of the Weierstrass Curve). [4] For any integer $j$ belonging to

$$
\left\{0,1, \ldots, N_{b}-2\right\}
$$

each natural integer $m$, and each word $\mathcal{M}_{m}$ of length $m$, let us consider the rectangle $\mathcal{R}_{j, m, \mathcal{M}_{m}}$, whose sides are parallel to the horizontal and vertical axes, of width:

$$
L_{m}=x\left(T_{\mathcal{M}_{m}}\left(P_{j+1}\right)\right)-x\left(T_{\mathcal{M}_{m}}\left(P_{j}\right)\right)=\frac{1}{\left(N_{b}-1\right) N_{b}^{m}}
$$

and height $\left|h_{j, m}\right|$, such that the points $T_{\mathcal{M}_{m}}\left(P_{j}\right)$ and $T_{\mathcal{M}_{m}}\left(P_{j+1}\right)$ are two vertices of this rectangle. We set:

$$
\eta_{\mathcal{W}}=2 \pi^{2}\left\{\frac{\left(2 N_{b}-1\right) \lambda\left(N_{b}^{2}-1\right)}{\left(N_{b}-1\right)^{2}(1-\lambda)\left(\lambda N_{b}^{2}-1\right)}+\frac{2 N_{b}}{\left(\lambda N_{b}^{2}-1\right)\left(\lambda N_{b}^{3}-1\right)}\right\} .
$$




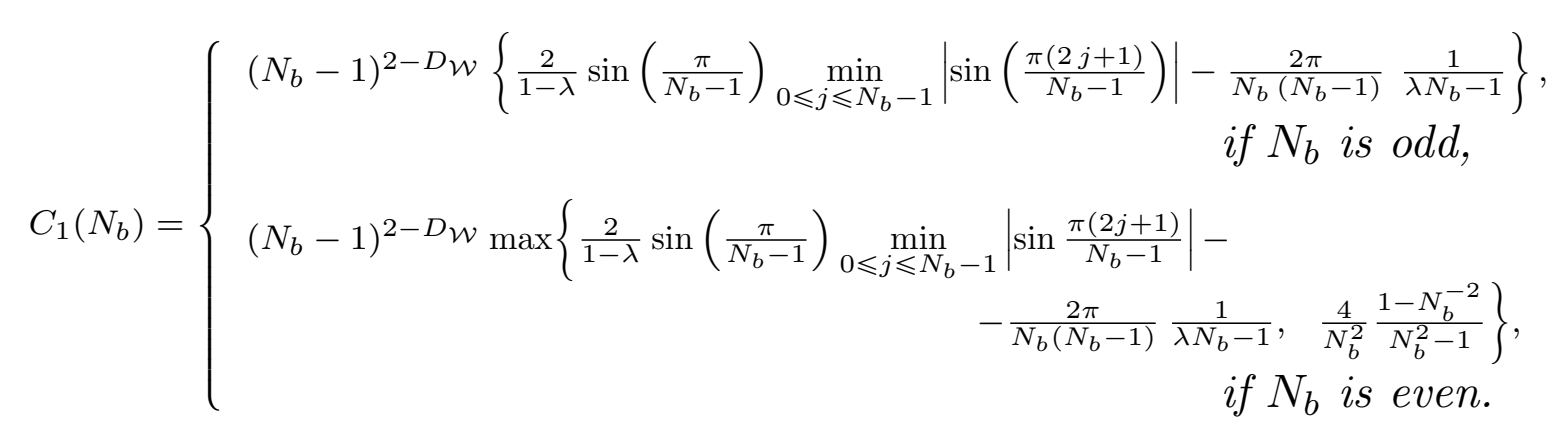

and:

$$
C_{2}\left(N_{b}\right)=\eta_{\mathcal{W}}\left(N_{b}-1\right)^{2-D_{\mathcal{W}}}
$$

Then:

$$
C_{1}\left(N_{b}\right) L_{m}^{2-D_{\mathcal{W}}} \leqslant\left|h_{j, m}\right| \leqslant C_{2}\left(N_{b}\right) L_{m}^{2-D_{\mathcal{W}}}
$$

Notation 1.17. Given a natural integer $m$, we set:

$$
h_{m}=L_{m}^{2-D_{\mathcal{W}}}=\frac{N_{b}^{\left(D_{\mathcal{W}}-2\right) m}}{\left(N_{b}-1\right)^{2-D_{\mathcal{W}}}} .
$$

Then the following inequality holds:

$$
h_{j m} \leqslant h_{m}
$$

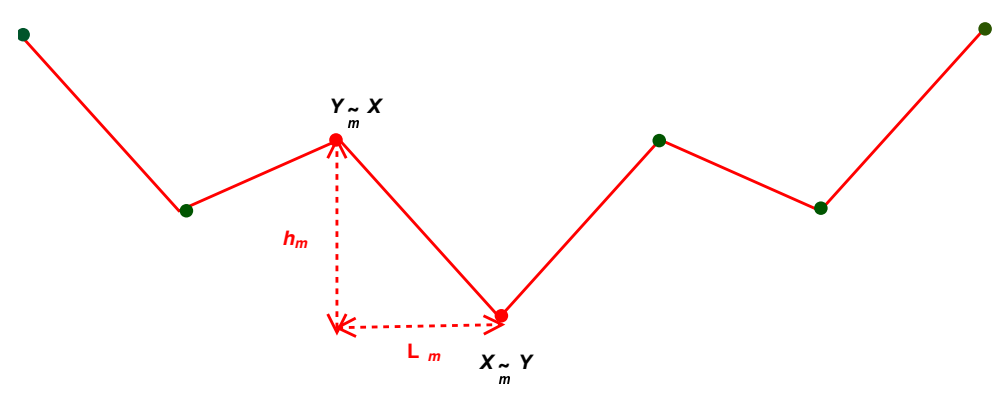

Corollary 1.18 (of Theorem 1.16). For any natural integer $m$, any integer $j$ belonging to $\left\{0,1, \ldots, N_{b}-2\right\}$, and each word $\mathcal{M}_{m+1}$ of length $m+1$, the two-dimensional Lebesgue measure

$$
\mu_{\mathcal{L}}\left(\mathcal{R}_{j, m+1, \mathcal{M}_{m+1}}\right)
$$

of the rectangle $\mathcal{R}_{j, m+1, \mathcal{M}_{m+1}}$, is such that, for any integer $k$ belonging to $\left\{0,1, \ldots, N_{b}-2\right\}$, any integer $\ell$ belonging to $\left\{0,1, \ldots, N_{b}-2\right\}$, and each word $\mathcal{M}_{m}$ of length $m$ :

$$
\mu_{\mathcal{L}}\left(\mathcal{R}_{j, m+1, \mathcal{M}_{m+1}}\right)<\mu_{\mathcal{L}}\left(\mathcal{R}_{\ell, m, \mathcal{M}_{m}}\right)
$$


Proof. Given a natural integer $m, j$ in $\left\{0,1, \ldots, N_{b}-2\right\}$, and a word $\mathcal{M}_{m+1}$ of length $m+1$, the two-dimensional Lebesgue measure of the rectangle $\mathcal{R}_{j, m+1, \mathcal{M}_{m+1}}$ can be obtained thanks to the values of the cartesian coordinates of the consecutive vertices $T_{\mathcal{M}_{m+1}}\left(P_{j}\right)$ and $T_{\mathcal{M}_{m+1}}\left(P_{j+1}\right)$ :

$$
\begin{aligned}
\mu_{\mathcal{L}}\left(\mathcal{R}_{j, m+1, \mathcal{M}_{m+1}}\right)=\left(x\left(T_{\mathcal{M}_{m+1}}\left(P_{j+1}\right)\right)-x\left(T_{\mathcal{M}_{m+1}}\left(P_{j}\right)\right)\right) \times \\
\times\left|y\left(T_{\mathcal{M}_{m+1}}\left(P_{j+1}\right)\right)-y\left(T_{\mathcal{M}_{m+1}}\left(P_{j}\right)\right)\right| .
\end{aligned}
$$

One may then write:

$$
T_{\mathcal{M}_{m+1}}=T_{k} \circ T_{\mathcal{M}_{m}}, \quad k \in\left\{0,1, \ldots, N_{b}-1\right\}
$$

where $\mathcal{M}_{m}$ is a word of length $m$. Thus, due to:

$$
\begin{gathered}
y\left(T_{\mathcal{M}_{m+1}}\left(P_{j+1}\right)\right)=\lambda y\left(T_{\mathcal{M}_{m}}\left(P_{j+1}\right)\right)+\cos \left(2 \pi\left(\frac{x\left(T_{\mathcal{M}_{m+1}}\left(P_{j+1}\right)\right)+k}{N_{b}}\right)\right) \\
y\left(T_{\mathcal{M}_{m+1}}\left(P_{j}\right)\right)=\lambda y\left(T_{\mathcal{M}_{m}}\left(P_{j}\right)\right)+\cos \left(2 \pi\left(\frac{x\left(T_{\mathcal{M}_{m+1}}\left(P_{j}\right)\right)+k}{N_{b}}\right)\right)
\end{gathered}
$$

and:

$$
x\left(T_{\mathcal{M}_{m}}\left(P_{j+1}\right)\right)-x\left(T_{\mathcal{M}_{m}}\left(P_{j}\right)\right)=L_{m} \leqslant\left|y\left(T_{\mathcal{M}_{m}}\left(P_{j+1}\right)\right)-y\left(T_{\mathcal{M}_{m}}\left(P_{j}\right)\right)\right|
$$

one has:

$$
\begin{aligned}
\left|y\left(T_{\mathcal{M}_{m+1}}\left(P_{j+1}\right)\right)-y\left(T_{\mathcal{M}_{m+1}}\left(P_{j}\right)\right)\right| \leqslant & \\
& \leqslant \lambda\left|y\left(T_{\mathcal{M}_{m}}\left(P_{j+1}\right)\right)-y\left(T_{\mathcal{M}_{m}}\left(P_{j}\right)\right)\right|+ \\
& \quad+\frac{2 \pi}{N_{b}}\left|x\left(T_{\mathcal{M}_{m}}\left(P_{j+1}\right)\right)-x\left(T_{\mathcal{M}_{m}}\left(P_{j}\right)\right)\right| \\
& \leqslant \lambda\left|y\left(T_{\mathcal{M}_{m}}\left(P_{j+1}\right)\right)-y\left(T_{\mathcal{M}_{m}}\left(P_{j}\right)\right)\right|+\frac{2 \pi}{N_{b}} L_{m} \\
& \leqslant\left(\lambda+\frac{2 \pi}{N_{b}}\right)\left|y\left(T_{\mathcal{M}_{m}}\left(P_{j+1}\right)\right)-y\left(T_{\mathcal{M}_{m}}\left(P_{j}\right)\right)\right|
\end{aligned}
$$

which yields:

$$
\begin{aligned}
\mu_{\mathcal{L}}\left(\mathcal{R}_{j, m+1, \mathcal{M}_{m+1}}\right) & =\frac{L_{m}}{N_{b}} \times\left|y\left(T_{\mathcal{M}_{m+1}}\left(P_{j+1}\right)\right)-y\left(T_{\mathcal{M}_{m+1}}\left(P_{j}\right)\right)\right| \\
& \leqslant \frac{L_{m}}{N_{b}} \times\left\{\lambda\left|y\left(T_{\mathcal{M}_{m}}\left(P_{j+1}\right)\right)-y\left(T_{\mathcal{M}_{m}}\left(P_{j}\right)\right)\right|+\frac{2 \pi}{N_{b}} L_{m}\right\} \\
& \leqslant \frac{L_{m}}{N_{b}} \times\left(\lambda+\frac{2 \pi}{N_{b}}\right)\left|y\left(T_{\mathcal{M}_{m}}\left(P_{j+1}\right)\right)-y\left(T_{\mathcal{M}_{m}}\left(P_{j}\right)\right)\right| .
\end{aligned}
$$


Due to the symmetric roles played by the integers $j$ and $\ell$, one has just to prove the result for $j=\ell$. Since:

$$
\mu_{\mathcal{L}}\left(\mathcal{R}_{j, m, \mathcal{M}_{m}}\right)=L_{m} \times\left|y\left(T_{\mathcal{M}_{m}}\left(P_{j+1}\right)\right)-y\left(T_{\mathcal{M}_{m}}\left(P_{j}\right)\right)\right|
$$

and, due to $N_{b} \geqslant 3$, we get that

$$
\frac{1}{N_{b}}\left(\lambda+\frac{2 \pi}{N_{b}}\right)-1=\frac{1}{N_{b}^{2}}\{\underbrace{\lambda N_{b}}_{<1}+2 \pi-N_{b}^{2}\}<0
$$

which yield the expected result.

\section{A SPECIFIC CLASS OF I.F.S.}

Weierstrass-type functions have been previously studied, but under the Hausdorff dimension point of view. One may refer, for instance, to the study by B. R. Hunt [7], where the author considers functions defined, for any real number $x$, by:

$$
W_{\Theta}(x)=\sum_{n=0}^{+\infty} a_{n} g\left(b_{n} x+\theta_{n}\right)
$$

where $\sum a_{n}$ is a positive and convergent series, $\left(b_{n}\right)_{n \in \mathbb{N}}$ a positive and increasing sequence, $\Theta=\left(\theta_{n}\right)_{n \in \mathbb{N}}$ a uniformly distribed sequence of numbers each belonging to $[0,1]$, and playing the part of arbitrary phases, $g$ being a Lispchitz and 1-periodic function.

In the case where the following assumptions are satisfied:

(i) there exist two strictly positive real numbers $\rho$ and $\sigma$ such that:

$$
1<\rho<\sigma, \quad \forall n \in \mathbb{N}: \quad \rho b_{n} \leqslant b_{n+1} \leqslant \sigma b_{n}
$$

(ii) there exists $D$ in $] 1,2[$ such that:

$$
\lim _{n \rightarrow+\infty} \frac{\ln a_{n}}{\ln b_{n}}=D-2
$$

(iii) there exist a positive integer $p$, a strictly positive real constant $M$, a constant $\ell$ in $(0,1)$, such that for all $\delta$ in $\left[\frac{\ell}{\sigma^{p}}, \ell\right]$, and for any real number $x$ chosen randomly according to a uniform distribution on $[0,1]$, the density function of:

$$
x \mapsto g(x+\delta)-g(x)
$$

has a $L^{\frac{p}{p-1}}$ norm at most equal to $M$.

B. R. Hunt [7] shows that for almost every $\Theta$ in $[0,1]^{\infty}$, the graph of $W_{\Theta}$ has Hausdorff dimension $D$. It happens that in the case of such functions, the Hausdorff dimension is equal to the box-dimension.

Yet, as concerns the lower bound estimate required to obtain the explicit value of the Hausdorff/box dimension, the author calls for strictly positive 
constants $K$ and $K^{\prime}$ which, as in existing earlier works, are not given explicitely (see, in the Hunt study, [7, section 3., page 798]). Moreover, no relation is made with the non-differentiability of such functions.

One may also note that such functions cannot be described by means of a finite iterated function systems, which does not allow any use of the Gluing Lemma.

In addition, the fact that the author considers, very generally, Lispchitz functions $g$ is not specifically justified. It is all the more interesting as evoked in the above since, if the functions $g$ were contractant ones, one falls back more easily on classical configurations. In fact, one may just consider the limit case of functions satisfying a Lipschitz condition with a Lipschitz constant of value 1 .

What seemed of interest to us was to generalize our results to, indeed, a class of Weierstrass-type functions, but defined through an iterated function system which would bear analogous properties of the maps $T_{i}, 0 \leqslant i \leqslant N_{b}-$ 1. First, the box-dimension can be obtained rather simply, without calling for theoretical background in dynamic systems theory, just by applying a similar method as in [4]. Then, one can also simply prove the nondifferentiability of such functions, as in [5].

Notation 2.1. In the sequel:

(i) $N$ is a strictly positive integer, greater than 2;

(ii) $T$ and $M$ are strictly positive real numbers;

(iii) $\left(\alpha_{i}\right)_{0 \leqslant i \leqslant N-1} \in\{0, \cdots, N-1\}^{N}$ and $\left(\beta_{i}\right)_{0 \leqslant i \leqslant N-1} \in\{0, \ldots, N-1\}^{N}$ are ordered sets of positive integers:

$$
\forall i \in\{0, \ldots, N-2\}: \quad \alpha_{i} \leqslant \alpha_{i+1}, \quad \beta_{i} \leqslant \beta_{i+1}
$$

(iv) $\psi$ is a T-periodic, bounded function from $\mathbb{R}$ to $\mathbb{R}$ satisfying a Lipschitz condition;

(v) $r_{y}$ is a real number such that:

$$
0<r_{y}<1, \quad r_{y} N>1 .
$$

(vi) We set:

$$
r_{x}=\frac{1}{N} .
$$

(vii) $\left\{\phi_{0}, \ldots, \phi_{N-1}\right\}$ and $\left\{\varphi_{0}, \ldots, \varphi_{N-1}\right\}$ are sets of affine contractive maps from $\mathbb{R}$ to $\mathbb{R}$, of respective ratios $r_{x}$ and $r_{y}$, defined, for any integer $i$ of $\{0, \ldots, N-1\}$, and for any real number $x$ :

$$
\phi_{i}(x)=r_{x}\left(x+\alpha_{i}\right), \quad \varphi_{i}(x)=r_{y}\left(x+\beta_{i}\right) .
$$


(viii) We denote by $\left\{\psi_{0}, \ldots, \psi_{N-1}\right\}$ the set of maps from $\mathbb{R}$ to $\mathbb{R}$ such that, for any integer $i$ of $\{0, \ldots, N-1\}$ :

$$
\psi_{i}=\psi \circ \phi_{i} \text {. }
$$

Notation 2.2. We introduce the set of maps from $\mathbb{R}^{2}$ to $\mathbb{R}^{2}$

$$
\left\{\widetilde{T}_{0}, \ldots, \widetilde{T}_{N-1}\right\}
$$

such that, for any integer $i$ of $\{0, \ldots, N-1\}$, and any $(x, y)$ of $\mathbb{R}^{2}$ :

$$
\widetilde{T}_{i}(x, y)=\left(\phi_{i}(x), \varphi_{i}(y)+\psi_{i}(x)\right) \text {. }
$$

Definition 2.3. We introduce the $\mathcal{W}$-type function, defined, for any real number $x$, by:

$$
\widetilde{\mathcal{W}}(x)=\sum_{n=0}^{+\infty} r_{y}^{n} \psi\left(T N^{n} x\right)
$$

Property 2.4. For any real number $x$, the series:

$$
\widetilde{\mathcal{W}}(x)=\sum_{n=0}^{+\infty} r_{y}^{n} \psi\left(T N^{n} x\right)
$$

is convergent

Proof. One may simply note that for any real number $x$ :

$$
\left|r_{y}^{n} \psi\left(T N^{n} x\right)\right| \lesssim r_{y}^{n} \sup _{t \in \mathbb{R}}|\psi(t)|
$$

which yields the expected result, since $\sum_{n=0}^{+\infty} r_{y}^{n}$ is a geometric convergent series.

Definition 2.5. We will call $\mathcal{W}$-type curve the restriction to $[0, T) \times \mathbb{R}$, of the graph of the $\mathcal{W}$-type function, and denote it by $\Gamma_{\widetilde{\mathcal{W}}}$.

2.6. Theoretical study. We place ourselves, in the following, in the euclidian plane of dimension 2, referred to a direct orthonormal frame. The usual Cartesian coordinates are $(x, y)$.

Property 2.7. For any integer $i$ of $\{0, \ldots, N-1\}$, the map $\widetilde{T}_{i}$ admits a fixed point, that we will denote by $\widetilde{P}_{i}$ :

$$
\widetilde{P}_{i}=\left(\frac{\alpha_{i}}{N-1}, \frac{\beta_{i}}{1-r_{y}}+\frac{1}{1-r_{y}} \psi_{i}\left(\frac{\alpha_{i}}{N-1}\right)\right)
$$

Lemma 2.8. For any integer $i$ belonging to $\{0, \ldots, N-1\}$, the map $T_{i}$ is a bijection of the Weierstrass-type curve on $\mathbb{R}$. 
Proof. Let us consider $i \in\{0, \ldots, N\}$, a point $(y, \mathcal{W}(y))$ of $\Gamma_{\widetilde{\mathcal{W}}}$, and let us look for a real number $x$ such that:

$$
T_{i}(x, \widetilde{\mathcal{W}}(x))=(y, \widetilde{\mathcal{W}}(y))
$$

One has:

$$
y=\phi_{i}(x)=r_{x}\left(x+\alpha_{i}\right)
$$

which yields:

$$
x=r_{x}^{-1} y-\alpha_{i} .
$$

This enables one to obtain:

$$
\begin{aligned}
\widetilde{\mathcal{W}}(x)=\mathcal{W}\left(r_{x}^{-1} y-\alpha_{i}\right) & =\sum_{n=0}^{+\infty} r_{y}^{n} \psi\left(T N^{n+1} y-T \alpha_{i} N^{n} i\right)= \\
& =\sum_{n=0}^{+\infty} r_{y}^{n} \psi\left(T N^{n+1} y\right)
\end{aligned}
$$

due to the $T$-periodicity of the function $\psi$, which leads to:

$$
\psi\left(T N^{n+1} y-T \alpha_{i} N^{n} i\right)=\psi\left(T N^{n+1} y\right)
$$

since $\alpha_{i}, N$ and $i$ are integers. Also:

$$
\begin{aligned}
\widetilde{T}_{i}(x, \widetilde{\mathcal{W}}(x)) & =\left(\phi_{i}\left(r_{x}^{-1} y-\alpha_{i}\right), \varphi_{i}(\widetilde{\mathcal{W}}(x))+\psi_{i}\left(r_{x}^{-1} y-\alpha_{i}\right)\right) \\
& =\left(y, r_{y} \sum_{n=0}^{+\infty} r_{y}^{n} \psi\left(T N^{n+1} y\right)+\psi(T y)\right) \\
& =\left(y, \sum_{n=0}^{+\infty} r_{y}^{n+1} \psi\left(T N^{n+1} y\right)+\psi(T y)\right) \\
& =\left(y, \sum_{n=0}^{+\infty} r_{y}^{n} \psi\left(T N^{n} y\right)\right) \\
& =(y, \widetilde{\mathcal{W}}(y)) .
\end{aligned}
$$

There exists thus a unique real number $x$ such that:

$$
T_{i}(x, \widetilde{\mathcal{W}}(x))=(y, \widetilde{\mathcal{W}}(y))
$$

Lemms is completed.

Theorem 2.9 (An upper bound and a lower bound for the box-dimension of the Weierstrass-type Curve). For any $j \in\{0,1, \ldots, N-2\}$, each natural integer $m$, and each word $\mathcal{M}$ of length $m$, let us consider the rectangle, whose sides are parallel to the horizontal and vertical axes, of width:

$$
L_{m}=x\left(T_{\mathcal{M}}\left(P_{j+1}\right)\right)-x\left(T_{\mathcal{M}}\left(P_{j}\right)\right)=r_{x}^{m}
$$


and height $\left|h_{j, m}\right|$, such that the points $T_{\mathcal{M}}\left(P_{j}\right)$ and $T_{\mathcal{M}}\left(P_{j+1}\right)$ are two vertices of this rectangle. We set:

$$
\begin{array}{r}
C_{1}(N)=\frac{1}{1-r_{y}} \min _{0 \leqslant j \leqslant N-1}\left\{\left(\beta_{i+1}-\beta_{i}\right)+\left\{\psi_{i+1}\left(\frac{\alpha_{i+1}}{N-1}\right)-\psi_{i}\left(\frac{\alpha_{i}}{N-1}\right)\right\}\right\}- \\
-\frac{r_{x}}{r_{y}} \frac{1}{1-\frac{r_{x}}{r_{y}}} \frac{\alpha_{j+1}-\alpha_{j}}{N-1}
\end{array}
$$

and:

$$
C_{2}(N)=\frac{\left|\beta_{j+1}-\beta_{j}\right|}{1-r_{y}}+\frac{1}{1-r_{y}}\left|\frac{\alpha_{j+1}}{N-1}-\frac{\alpha_{j}}{N-1}\right|+\frac{\left|\alpha_{j+1}-\alpha_{j}\right|}{N\left(r_{y}-r_{x}\right)}
$$

If:

$$
C_{1}(N) \geqslant 0
$$

one has:

$$
C_{1}(N) L_{m}^{2-D_{\widetilde{\mathcal{W}}}} \leqslant\left|h_{j, m}\right| \leqslant C_{2}(N) L_{m}^{2-D_{\widetilde{\mathcal{W}}}}
$$

where:

$$
D_{\widetilde{\mathcal{W}}}=2+\frac{\ln r_{y}}{\ln N}
$$

which yields the fractal character of the Weierstrass-type Curve, the boxdimension of which is then $D_{\widetilde{\mathcal{W}}}$.

Proof. The proof is obtained as in [4]. It is based on the fact that, given a strictly positive integer $m$, and two points $X$ and $Y$ of $V_{m}$ such that:

$$
X \underset{m}{\sim} Y
$$

there exists a word $\mathcal{M}$ of length $|\mathcal{M}|=m$, on the graph $\Gamma_{\widetilde{\mathcal{W}}}$, and an integer $j$ of $\{0, \ldots, N-2\}^{2}$, such that:

$$
X=\widetilde{T}_{\mathcal{M}}\left(P_{j}\right), \quad Y=\widetilde{T}_{\mathcal{M}}\left(P_{j+1}\right) .
$$

By writing $\widetilde{T}_{\mathcal{M}}$ under the form:

$$
\widetilde{T}_{\mathcal{M}}=\tilde{T}_{i_{m}} \circ \tilde{T}_{i_{m-1}} \circ \ldots \circ \tilde{T}_{i_{1}}
$$

where $\left(i_{1}, \ldots, i_{m}\right) \in\{0, \ldots, N-1\}^{m}$, one gets:

$$
x\left(\widetilde{T}_{\mathcal{M}}\left(P_{j}\right)\right)=r_{x}^{N} x_{j}+\sum_{k=1}^{m} r_{x}^{k} \alpha_{k}, \quad x\left(\widetilde{T}_{\mathcal{M}}\left(P_{j+1}\right)\right)=r_{x}^{N} x_{j+1}+\sum_{k=1}^{m} r_{x}^{k} \alpha_{k}
$$

and

$$
y\left(\widetilde{T}_{\mathcal{M}}\left(P_{j}\right)\right)=r_{y}^{m} y_{j}+\sum_{k=1}^{m} r_{y}^{m-k} \psi_{k}\left(r_{x}^{k} x_{j}+\sum_{\ell=0}^{k} r_{x}^{\ell} \alpha_{m-\ell}\right)
$$




$$
y\left(\widetilde{T}_{\mathcal{M}}\left(P_{j+1}\right)\right)=r_{y}^{m} y_{j+1}+\sum_{k=1}^{m} r_{y}^{m-k} \psi_{k}\left(r_{x}^{k} x_{j+1}+\sum_{\ell=0}^{k} r_{x}^{\ell} \alpha_{m-\ell}\right)
$$

This leads to

$$
\begin{aligned}
h_{j, m}-r_{y}^{m}\left(y_{j+1}-y_{j}\right)=\sum_{k=1}^{m} r_{y}^{m-k}\left\{\psi _ { i _ { k } } \left(r_{x}^{k} x_{j+1}\right.\right. & \left.+\sum_{\ell=0}^{k} r_{x}^{\ell} \alpha_{m-\ell}\right)- \\
& \left.-\psi_{i_{k}}\left(r_{x}^{k} x_{j}+\sum_{\ell=0}^{k} r_{x}^{\ell} \alpha_{m-\ell}\right)\right\},
\end{aligned}
$$

where

$$
r_{y}^{m}\left(y_{j+1}-y_{j}\right)=r_{y}^{m} \frac{\beta_{j+1}-\beta_{j}}{1-r_{y}}+\frac{r_{y}^{m}}{1-r_{y}}\left\{\psi_{j+1}\left(\frac{\alpha_{j+1}}{N-1}\right)-\psi_{j}\left(\frac{\alpha_{j}}{N-1}\right)\right\} .
$$

Since the maps $\psi_{i_{k}}, 1 \leqslant k \leqslant m$, satisfy a Lipschitz condition, with a Lipschitz constant equal to 1 , one has thus:

$$
\begin{aligned}
\left|y\left(T_{\mathcal{M}}\left(P_{j}\right)\right)-y\left(T_{\mathcal{M}}\left(P_{j+1}\right)\right)-r_{y}^{m}\left(y_{j+1}-y_{j}\right)\right| \leqslant & \\
& \leqslant \sum_{k=1}^{m} r_{y}^{m-k} r_{x}^{k}\left|x_{j+1}-x_{j}\right|=\sum_{k=1}^{m} r_{y}^{m-k} r_{x}^{k} \frac{\left|\alpha_{j+1}-\alpha_{j}\right|}{N-1}= \\
& =r_{y}^{m} \frac{r_{x}}{r_{y}} \frac{1-\frac{r_{x}^{m}}{r_{y}^{m}}}{1-\frac{r_{x}}{r_{y}}} \frac{\left|\alpha_{j+1}-\alpha_{j}\right|}{N-1} \leqslant r_{y}^{m} \frac{r_{x}}{r_{y}} \frac{1}{1-\frac{r_{x}}{r_{y}}} \frac{\left|\alpha_{j+1}-\alpha_{j}\right|}{N-1},
\end{aligned}
$$

which leads to

$$
\begin{aligned}
y\left(T_{\mathcal{M}}\left(P_{j}\right)\right)- & y\left(T_{\mathcal{M}}\left(P_{j+1}\right)\right) \geqslant r_{y}^{m} \frac{\beta_{j+1}-\beta_{j}}{1-r_{y}}+ \\
& +\frac{r_{y}^{m}}{1-r_{y}}\left\{\psi_{i+1}\left(\frac{\alpha_{i+1}}{N-1}\right)-\psi_{i}\left(\frac{\alpha_{i}}{N-1}\right)\right\}-r_{x} \frac{r_{y}^{m}-r_{x}^{m}}{r_{y}-r_{x}} \frac{\left|\alpha_{j+1}-\alpha_{j}\right|}{N-1}
\end{aligned}
$$

If

$\frac{1}{1-r_{y}} \min _{0 \leqslant j \leqslant N-1}\left\{\left(\beta_{j+1}-\beta_{j}\right)+\left\{\psi_{j+1}\left(\frac{\alpha_{j+1}}{N-1}\right)-\psi_{j}\left(\frac{\alpha_{j}}{N-1}\right)\right\}\right\}-$

$$
-\frac{r_{x}}{r_{y}} \frac{1}{1-\frac{r_{x}}{r_{y}}} \frac{\left|\alpha_{j+1}-\alpha_{j}\right|}{N-1} \geqslant 0
$$

due to the symmetric roles played by $T_{\mathcal{M}}\left(P_{j}\right)$ and $T_{\mathcal{M}}\left(P_{j+1}\right)$, one may only consider the case when

$$
\begin{aligned}
& y\left(T_{\mathcal{M}}\left(P_{j}\right)\right)-y\left(T_{\mathcal{M}}\left(P_{j+1}\right)\right) \geqslant r_{y}^{m} \frac{\beta_{j+1}-\beta_{j}}{1-r_{y}}+ \\
& \quad+\frac{r_{y}^{m}}{1-r_{y}}\left\{\psi_{i+1}\left(\frac{\alpha_{i+1}}{N-1}\right)-\psi_{i}\left(\frac{\alpha_{i}}{N-1}\right)\right\}-r_{y}^{m} \frac{r_{x}}{r_{y}} \frac{1}{1-\frac{r_{x}}{r_{y}}} \frac{\left|\alpha_{j+1}-\alpha_{j}\right|}{N-1} \geqslant 0 .
\end{aligned}
$$


which yields

$$
\begin{array}{r}
y\left(T_{\mathcal{M}}\left(P_{j}\right)\right)-y\left(T_{\mathcal{M}}\left(P_{j+1}\right)\right) \geqslant \\
\geqslant r_{y}^{m}\left\{\frac{1}{1-r_{y}} \min _{0 \leqslant j \leqslant N-1}\left\{\left(\beta_{i+1}-\beta_{i}\right)+\left\{\psi_{i+1}\left(\frac{\alpha_{i+1}}{N-1}\right)-\psi_{i}\left(\frac{\alpha_{i}}{N-1}\right)\right\}\right\}-\right. \\
\left.-\frac{r_{x}}{r_{y}} \frac{1}{1-\frac{r_{x}}{r_{y}}} \frac{\alpha_{j+1}-\alpha_{j}}{N-1}\right\} .
\end{array}
$$

The predominant term is thus

$$
r_{y}^{m}=e^{m\left(D_{\widetilde{\mathcal{W}}}-2\right) \ln N}=N^{m\left(D_{\widetilde{\mathcal{W}}}-2\right)}=L_{m}^{2-D_{\widetilde{\mathcal{W}}}}(N-1)^{2-D_{\widetilde{\mathcal{W}}}}
$$

One also has

$$
\begin{aligned}
\left|h_{j, m}\right| & \leqslant r_{y}^{m}\left|y_{j+1}-y_{j}\right|+ \\
& +\sum_{k=1}^{m} r_{y}^{m-k}\left|\psi_{i_{k}}\left(r_{x}^{k} x_{j+1}+\sum_{\ell=0}^{k} r_{x}^{\ell} \alpha_{m-\ell}\right)-\psi_{i_{k}}\left(r_{x}^{k} x_{j}+\sum_{\ell=0}^{k} r_{x}^{\ell} \alpha_{m-\ell}\right)\right| \\
& \leqslant r_{y}^{m} \frac{\left|\beta_{j+1}-\beta_{j}\right|}{1-r_{y}}+\frac{r_{y}^{m}}{1-r_{y}}\left|\psi_{j+1}\left(\frac{\alpha_{j+1}}{N-1}\right)-\psi_{j}\left(\frac{\alpha_{j}}{N-1}\right)\right| \\
& +\sum_{k=1}^{m} r_{y}^{m-k}\left|\psi_{i_{k}}\left(r_{x}^{k} x_{j+1}+\sum_{\ell=0}^{\ell} r_{x}^{\ell} \alpha_{m-\ell}\right)-\psi_{i_{k}}\left(r_{x}^{k} x_{j}+\sum_{\ell=0}^{k} r_{x}^{\ell} \alpha_{m-\ell}\right)\right| \\
& \leqslant r_{y}^{m} \frac{\left|\beta_{j+1}-\beta_{j}\right|}{1-r_{y}}+\frac{r_{y}^{m}}{1-r_{y}}\left|\frac{\alpha_{j+1}}{N-1}-\frac{\alpha_{j}}{N-1}\right|+\sum_{k=1}^{m} r_{y}^{m-k} r_{x}^{k}\left|x_{j+1}-x_{j}\right| \\
& =r_{y}^{m} \frac{\left|\beta_{j+1}-\beta_{j}\right|}{1-r_{y}}+\frac{r_{y}^{m}}{1-r_{y}}\left|\frac{\alpha_{j+1}}{N-1}-\frac{\alpha_{j}}{N-1}\right|+\left|x_{j+1}-x_{j}\right| r_{y}^{m} \frac{r_{x}}{r_{y}} \frac{r_{x}^{m}}{1-\frac{r_{x}}{r_{y}^{m}}} \\
& \leqslant r_{y}^{m} \frac{\left|\beta_{j+1}-\beta_{j}\right|}{1-r_{y}}+\frac{r_{y}^{m}}{1-r_{y}}\left|\frac{\alpha_{j+1}}{N-1}-\frac{\alpha_{j}}{N-1}\right|+\left|x_{j+1}-x_{j}\right| r_{x} \frac{r_{y}^{m}-r_{x}^{m}}{r_{y}-r_{x}} \\
& \leqslant r_{y}^{m} \frac{\left|\beta_{j+1}-\beta_{j}\right|}{1-r_{y}}+\frac{r_{y}^{m}}{1-r_{y}}\left|\frac{\alpha_{j+1}}{N-1}-\frac{\alpha_{j}}{N-1}\right|+\left|x_{j+1}-x_{j}\right| r_{x} \frac{r_{y}^{m}}{r_{y}-r_{x}} \\
& \leqslant \frac{\alpha_{j+1}}{N-1}-\frac{\alpha_{j}}{N-1} \mid+r_{y}^{m} \frac{\left|\alpha_{j+1}-\alpha_{j}\right|}{N\left(r_{y}-r_{x}\right)} .
\end{aligned}
$$

Since

$$
D_{\widetilde{\mathcal{W}}}=2+\frac{\ln r_{y}}{\ln N}, \quad \quad r_{y}=e^{\left(D_{\widetilde{\mathcal{W}}}-2\right) \ln N}=N^{\left(D_{\widetilde{\mathcal{W}}}-2\right)}
$$


one has thus:

$$
\left|h_{j, m}\right| \leqslant r_{y}^{m}\left\{\frac{\left|\beta_{j+1}-\beta_{j}\right|}{1-r_{y}}+\frac{1}{1-r_{y}}\left|\frac{\alpha_{j+1}}{N-1}-\frac{\alpha_{j}}{N-1}\right|+\frac{\left|\alpha_{j+1}-\alpha_{j}\right|}{N\left(r_{y}-r_{x}\right)}\right\} .
$$

Theorem 2.9 is proved.

Corollary 2.10. The $\mathcal{W}$-type functions are non-differentiable.

Proof. One has simply to use the analogous density property as in 1.11.

Given a natural integer $m$, and two points $X=(x, \widetilde{\mathcal{W}}(x)), Y=(y, \widetilde{\mathcal{W}}(y))$ of the pre-fractal graph $\Gamma_{\widetilde{\mathcal{W}}_{m}} \subset \Gamma_{\widetilde{\mathcal{W}}}$ such that:

$$
x \leqslant y, \quad X \underset{m}{\sim} Y
$$

one may write:

$$
X=\widetilde{T}_{\mathcal{M}_{m, j}}\left(P_{k}\right), \quad Y=\left(x+L_{m}, \mathcal{W}\left(x+L_{m}\right)\right)=\widetilde{T}_{\mathcal{M}_{m, j}}\left(P_{k+1}\right)
$$

where $\mathcal{M}_{m, j}, 0 \leqslant j \leqslant N^{m}-1$ is a word of length $m$, while $k$ denotes an integer of the set $\{0, \ldots, N-2\}$.

One may note that

$$
\left|x\left(\widetilde{T}_{\mathcal{M}_{m, j}}\left(P_{k}\right)\right)-x\left(\widetilde{T}_{\mathcal{M}_{m, j}}\left(P_{k+1}\right)\right)\right|=\frac{1}{N^{m}}=L_{m} \underset{m \rightarrow+\infty}{\longrightarrow} 0 .
$$

Thus

$$
\begin{aligned}
& \mid \widetilde{\mathcal{W}}\left(x\left(\widetilde{T}_{\mathcal{M}_{m, j}}\left(P_{k}\right)\right)\right)-\widetilde{\mathcal{W}}\left(x\left(\widetilde{T}_{\mathcal{M}_{m, j}}\left(P_{k+1}\right)\right)\right) \mid \geqslant C_{1}(N) L_{m}^{2-D_{\widetilde{\mathcal{W}}}}= \\
& \quad=C_{1}(N)\left|x\left(\widetilde{T}_{\mathcal{M}_{m, j}}\left(P_{k}\right)\right)-x\left(\widetilde{T}_{\mathcal{M}_{m, j}}\left(P_{k+1}\right)\right)\right|^{2-D_{\widetilde{\mathcal{W}}}}
\end{aligned}
$$

which leads to

$$
\begin{aligned}
&\left|\frac{\widetilde{\mathcal{W}}\left(x\left(\widetilde{T}_{\mathcal{M}_{m, j}}\left(P_{k}\right)\right)\right)-\widetilde{\mathcal{W}}\left(x\left(\widetilde{T}_{\mathcal{M}_{m, j}}\left(P_{k+1}\right)\right)\right)}{x\left(\widetilde{T}_{\mathcal{M}_{m, j}}\left(P_{k}\right)\right)-x\left(\widetilde{T}_{\mathcal{M}_{m, j}}\left(P_{k+1}\right)\right)}\right| \geqslant \\
& \geqslant C_{1}(N)\left|x\left(\widetilde{T}_{\mathcal{M}_{m, j}}\left(P_{k}\right)\right)-x\left(\widetilde{T}_{\mathcal{M}_{m, j}}\left(P_{k+1}\right)\right)\right|^{1-D_{\widetilde{\mathcal{W}}}}= \\
&=C_{1}(N) L_{m}^{1-D_{\widetilde{\mathcal{W}}}}
\end{aligned}
$$

where

$$
1-D_{\widetilde{\mathcal{W}}}=-1-\frac{\ln r_{y}}{\ln N}=-\frac{\ln \left(r_{y} N\right)}{\ln N}<0
$$

By passing to the limit when the integer $m$ tends towards infinity, one gets the non-differentiability expected result:

$$
\lim _{m \rightarrow+\infty}\left|\frac{\widetilde{\mathcal{W}}\left(x+L_{m}\right)-\widetilde{\mathcal{W}}(x)}{L_{m}}\right|=+\infty
$$


where:

$$
\lim _{m \rightarrow+\infty} L_{m}=0
$$

Corollary is completed.

\section{REFERENCES}

[1] Krzysztof Barański, Balázs Bárány, Julia Romanowska. On the dimension of the graph of the classical Weierstrass function. Adv. Math., 265:32-59, 2014, doi: $10.1016 /$ j.aim.2014.07.033.

[2] M. F. Barnsley, S. Demko. Iterated function systems and the global construction of fractals. Proc. Roy. Soc. London Ser. A, 399(1817):243-275, 1985, doi: $10.1098 /$ rspa. 1985.0057.

[3] A. S. Besicovitch, H. D. Ursell. Sets of fractional dimensions (V): on dimensional numbers of some continuous curves. J. London Math. Soc., s1-12(1):18-25, 1937, doi: $10.1112 / \mathrm{jlms} / \mathrm{s} 1-12.45 .18$.

[4] Claire David. Bypassing dynamical systems: a simple way to get the box-counting dimension of the graph of the Weierstrass function. Proc. Int. Geom. Cent., 11(2):5368, 2018, doi: 10.15673/tmgc.v11i2.1028.

[5] Claire David. Wandering across the Weierstrass function, while revisiting its properties. To appear, 2019.

[6] Robert L. Devaney. An introduction to chaotic dynamical systems. Studies in Nonlinearity. Westview Press, Boulder, CO, 2003. Reprint of the second (1989) edition.

[7] Brian R. Hunt. The Hausdorff dimension of graphs of Weierstrass functions. Proc. Amer. Math. Soc., 126(3):791-800, 1998, doi: 10.1090/S0002-9939-98-04387-1.

[8] John E. Hutchinson. Fractals and self-similarity. Indiana Univ. Math. J., 30(5):713747, 1981, doi: 10.1512/iumj.1981.30.30055.

[9] Gerhard Keller. A simpler proof for the dimension of the graph of the classical Weierstrass function. Ann. Inst. Henri Poincaré Probab. Stat., 53(1):169-181, 2017, doi: $10.1214 / 15-A I H P 711$.

[10] Jun Kigami. A harmonic calculus on the Sierpiński spaces. Japan J. Appl. Math., 6(2):259-290, 1989, doi: 10.1007/BF03167882.

[11] Benoit B. Mandelbrot. Fractals: form, chance, and dimension. W. H. Freeman and Co., San Francisco, Calif., revised edition, 1977. Translated from the French.

[12] Benoit B. Mandelbrot. The fractal geometry of nature. W. H. Freeman and Co., San Francisco, Calif., 1982. Schriftenreihe für den Referenten.

[13] K. Weierstrass. Über kontinuierliche Funktionen eines reellen arguments, die für keinen Wert des letzteren einen bestimmten Differentialquotienten besitzen. Journal für die reine und angewandte Mathematik, 79:29-31, 1875.

Claire David

Sorbonne Université, CNRS, Laboratoire Jacques-Louis Lions, 4, Place Jussieu 75005, Paris, France

Email: Claire.David@upmc.fr

ORCID: orcid.org/0000-0002-4729-0733 